may have been confined by the adherent pericardium, and the mouth of it may have closed spontaneously. ${ }^{4}$

\section{CONCLUSIONS}

This case demonstrated that it was feasible to reconstruct a ventricular septum using a double Dacron patch to replace the immovable calcification, and the short-term result was satisfied. More cases are needed to prove the safety and long-term results of the unusual method.

\section{References}

1. Lee BK, Atwood JE. Calcified left ventricular aneurysm. NEngl J Med. 2003;348:918

2. Uz O, Kardesoglu E, Yalcin M, Cebeci BS. Calcified left ventricular aneurysm with a giant mural thrombus. Heart. 2009;95:993.

3. Harrison-Gómez C, Harrison-Ragle A, Arceo-Navarro A. A ring in the heart calcified left ventricular aneurysm. Circulation. 2007;115:e376-7.

4. Raudkivi PJ, Smyllie J, Conway N, Ross JK. Rupture of a calcified true left ventricular aneurysm: echocardiographic diagnosis and successful repair. Eur J Cardiothorac Surg. 1989;3:81-4.

5. Kodama K, Jo T, Fujiwara Y, et al. Clinical characteristics of postinfarction left ventricular aneurysm with extensive calcification. Nippon Ronen Igakkai Zasshi. 1992;29:565-73.

\title{
Implantation of a centrifugal pump as a left ventricular assist device through a novel, minimized approach: Upper hemisternotomy combined with anterolateral thoracotomy
}

\author{
Jan D. Schmitto, MD, ${ }^{\mathrm{a}}$ Ulrich Molitoris, MD, ${ }^{\mathrm{b}}$ Axel Haverich, MD, ${ }^{\mathrm{a}}$ and Martin Strueber, MD, ${ }^{\mathrm{a}}$ \\ Hannover, Germany
}

The role and the use of ventricular assist devices (VADs) for the treatment of chronic heart failure are increasing tremendously worldwide. The VAD-related success is mainly based on 2 important factors: (1) novel technologies and (2) increased surgical experience leading to lower complication rates and better outcomes.

Mechanical cardiac circulatory support with the novel HeartWare ventricular assist device (HVAD; HeartWare International, Inc, Framingham, Mass) has become an established therapy for left ventricular (LV) failure. ${ }^{1-3}$ Within the past years more than 150 cardiac assist devices were implanted at our institution, and the total number of VAD implantations continues to increase steadily. While complication rates decrease, survivals increase and the longterm outcomes have improved significantly over the years.

Herein we report the case of a male patient with chronic heart failure (New York Heart Association class III-IV) secondary to dilated cardiomyopathy in whom we successfully implanted a centrifugal blood pump (HeartWare) as an LV

\footnotetext{
From the Department of Cardiac, Thoracic, Transplantation and Vascular Surgery ${ }^{\mathrm{a}}$ and the Department of Anaesthesiology and Intensive Care, ${ }^{\mathrm{b}}$ Hannover Medical School, Hannover, Germany.

Disclosures: Authors have nothing to disclose with regard to commercial support.

Received for publication June 7, 2011; revisions received July 12, 2011; accepted for publication July 22, 2011; available ahead of print Sept 8, 2011.

Address for reprints: Jan D. Schmitto, MD, PhD, Department of Cardiac, Transplantation, Thoracic and Vascular Surgery, Hannover Medical School, Carl-NeubergStrasse 1, 30625 Hannover, Germany (E-mail: schmitto.jan@mh-hannover.de). J Thorac Cardiovasc Surg 2012;143:511-3 $0022-5223 / \$ 36.00$

Copyright (c) 2012 by The American Association for Thoracic Surgery doi:10.1016/j.jtcvs.2011.07.046
}

assist device (LVAD). We used a novel implantation technique (upper hemisternotomy combined with anterolateral thoracotomy) to reduce complication rates (minimize trauma, reduce bleeding complications, and avoid right heart failure).

\section{CLINICAL SUMMARY}

A 41-year-old male patient was admitted with chronic heart failure resulting from dilative cardiomyopathy (New York Heart Association class III-IV). Echocardiography revealed an ejection fraction of $15 \%$, cardiac index was measured at $1.5 \mathrm{~L} \cdot \mathrm{min}^{-1} \cdot \mathrm{m}^{-2}$, central venous saturation decreased to $35 \%$, and the patient reported having shortness of breath at rest. Therefore, catecholamine therapy was initiated with dobutamine. Additionally, levosimendan therapy was started in the intensive care unit. However, cardiac output still remained low $(<4 \mathrm{~L} / \mathrm{min})$, the patient became clinically instable, and the only realistic option was the surgical implantation of an LVAD. Therefore, the patient underwent urgent VAD implantation using a novel, minimized surgical approach.

The first step, transfemoral venous cannulation, was performed through the right femoral vein by the Seldinger technique. Second, upper hemisternotomy was used to implant the arterial cannula into the ascending aorta for extracorporeal circulatory support. Then, left anterolateral thoracotomy was performed to implant the VAD inflow cannula in the LV apex. Last, an end-toside anastomosis of the VAD outflow cannula to the ascending aorta was performed through the upper hemisternotomy. 


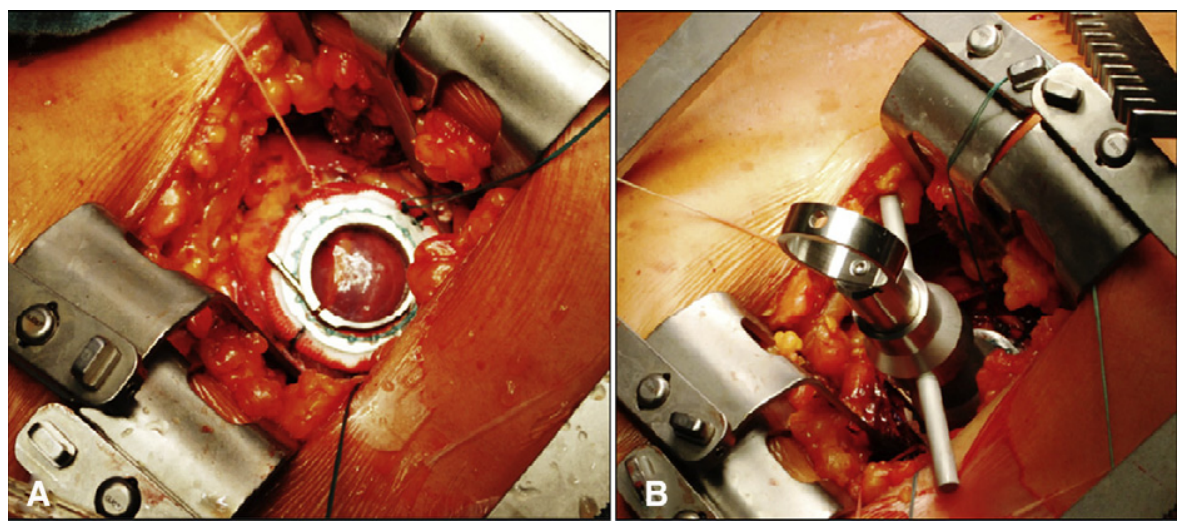

FIGURE 1. A, Sewing ring of the HeartWare ventricular assist device anastomosed to the left ventricular apex through left anterolateral thoracotomy. B, Preparation for implantation of the ventricular assist device into the left ventricular apex.

\section{OPERATIVE PROCEDURE}

Cardiopulmonary bypass was established by venous cannulation into the right femoral vein and arterial cannulation into the ascending aorta via an upper hemisternotomy. The pericardium remained mainly closed (opened only anterior to the ascending aorta), thereby leading to further stabilization of right ventricular (RV) function including avoidance of RV dilation during the surgical procedure, especially during the start-up of the LVAD.

Then, an anterolateral thoracotomy was performed and an epicardial HVAD sewing ring was implanted on the LV apex using a 2-0-running Prolene polypropylene suture (Ethicon, Inc, Somerville, NJ) (Figure 1, A). After the HVAD ring had been sewn to the apex, sutures and the ring were sealed with BioGlue surgical adhesive (CryoLife, Inc, Kennesaw, Ga). Afterward, the HVAD pump was placed through the sewing ring into the LV apex (Figure 1,B). The outflow vascular graft was tunneled from the thoracotomy to the upper hemisternotomy through the pericardium and was anastomosed end-to-side to the ascending aorta (Figure 2, A). The driveline was placed in the sheath of the rectus muscle in the umbilical direction and then subcutaneously to the left upper quadrant. After deairing through a stitch incision of the outflow graft, the LVAD pump was started in situ (Figure 2, B). Pump speed was gradually increased during weaning from extracorporeal circulation. A pump flow of $5.5 \mathrm{~L}$ was achieved with the LVAD running at $3000 \mathrm{rpm}$.

After closure of the 2 small wounds, the patient was transferred to the intensive care unit with minimal catecholamine support and sufficient VAD flow. After successful respiratory weaning, he was extubated on postoperative day 1 , always displaying stable hemodynamics.

His further clinical stay at our department was completely uneventful. Currently, the patient is awaiting cardiac transplantation in a fully ambulatory state.

In summary, this case describes a novel technique for LVAD implantation (HVAD centrifugal pump) using a minimized approach: upper hemisternotomy in combination with left anterolateral thoracotomy.

\section{DISCUSSION}

This novel, minimized LVAD implantation technique (HVAD centrifugal pump) through an upper hemisternotomy combined with anterolateral thoracotomy is associated with several advantages:

First, the surgical approach is minimized compared with a full sternotomy, thereby minimizing surgical trauma and leading to reduced complication rates (eg, less postoperative bleeding, reduced need for transfusion products, less pain, less use of anticoagulation drugs, and lower costs). ${ }^{4}$

Second, despite the fact that other VAD surgeons recommended HVAD placement on the diaphragmatic surface of the $\mathrm{LV},{ }^{3}$ our experience has confirmed that the VAD placement with our novel technique results in an optimal and stable position of the inflow cannula through an anterolateral approach to the LV apex (Figure 2,C). Our novel approach results in protection against inflow obstruction and endocardial contact, therefore avoiding arrhythmias.

Third, the pericardium remained mainly closed, thereby leading to further stabilization of RV function including avoidance of RV dilation during the procedure, especially during the start-up of the LVAD.

The limitations of this novel approach include the following: (1) Visual control of cardiac contractile function, especially of the RV, is reduced; (2) the minimized surgical approach is technically more difficult to perform than a full sternotomy; and (3) estimation of the exact length of the outflow graft is more challenging than in the conventional technique.

\section{CONCLUSIONS}

Surgical implantation of an LVAD through minimized surgical approaches is proven to be feasible ${ }^{5}$ and 

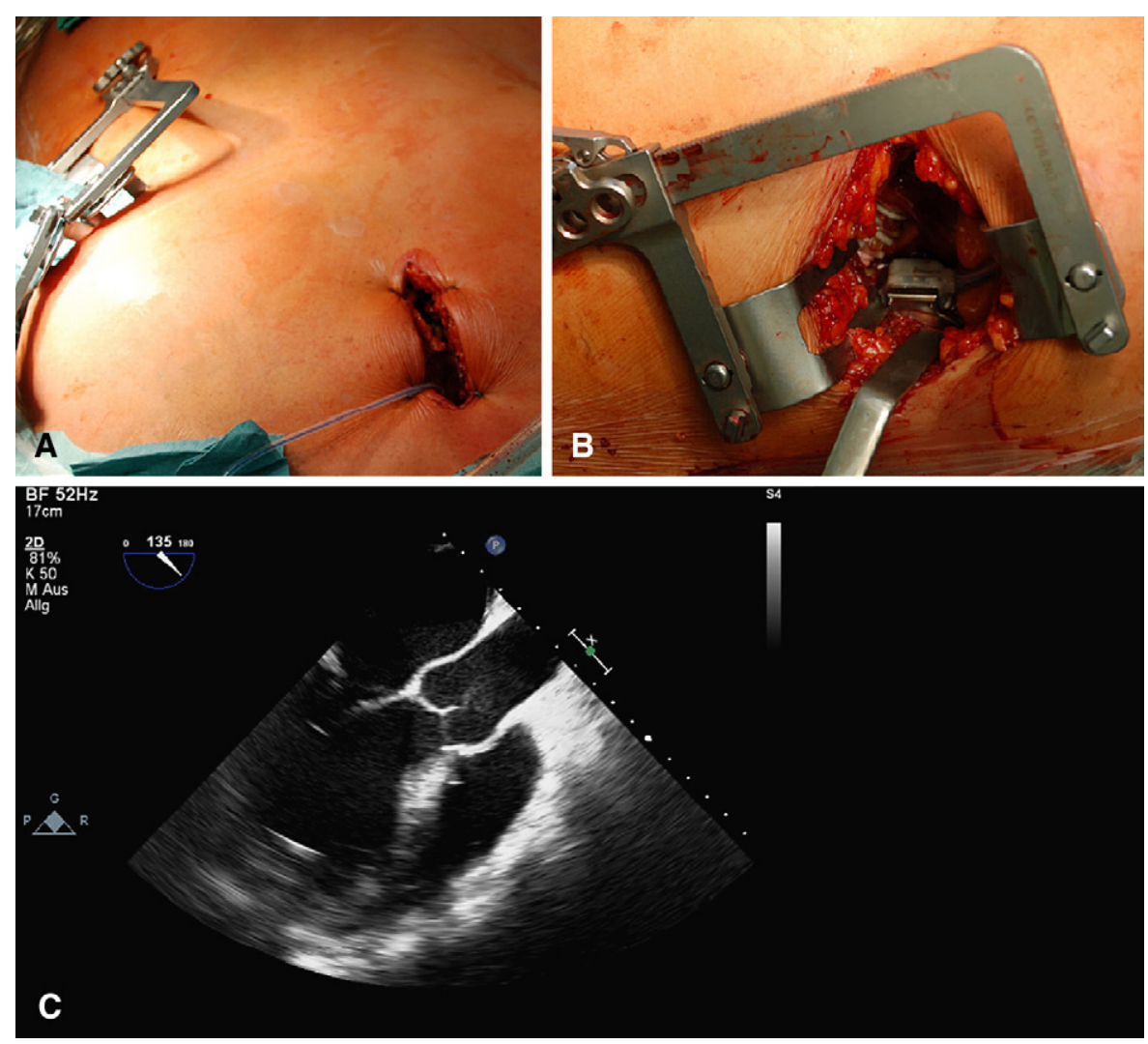

FIGURE 2. A, Upper hemisternotomy to perform arterial cannulation to the ascending aorta as well as for implantation of the outflow cannula of the centrifugal pump. B, In situ placement of the HeartWare ventricular assist device pump through the anterolateral thoracotomy. C, Postoperative echocardiography showing the stable central placement of the inflow cannula of the left ventricular assist device into the left ventricle through an anterolateral approach.

associated with several positive aspects. ${ }^{4}$ Most important, surgical trauma is minimized (as already proven for other minimally invasive procedures ${ }^{4}$ ). This may lead to reduced complication rates, including fewer postoperative bleeding complications, reduced need for transfusion products, less pain, less use of anticoagulation drugs, and lower costs. ${ }^{4}$ Additionally, we believe that the occurrence of right heart failure could probably be reduced by this approach because the pericardium remains closed.

However, this novel procedure is technically more difficult to perform than is full sternotomy. Therefore, such minimized approaches should be performed only at specialized centers where cardiac surgeons are widely experienced in the standard technique for LVAD implantation and diligent in evaluating their results to ensure the highest quality of VAD surgery.

\section{References}

1. Strueber M, O'Driscoll G, Jansz P, Khaghani A, Levy WC, et al. HeartWare Investigators. Multicenter evaluation of an intrapericardial left ventricular assist system. J Am Coll Cardiol. 2011;57:1375-82.

2. Strueber M, Meyer AL, Malehsa D, Haverich A. Successful use of the HeartWare HVAD rotary blood pump for biventricular support. J Thorac Cardiovasc Surg. 2010;140:936-7.

3. Gregoric ID, Cohn WE, Frazier OH. Diaphragmatic implantation of the HeartWare ventricular assist device. J Heart Lung Transplant. 2011;30:467-70. Epub 2011 Jan 6 .

4. Schmitto JD, Mokashi SA, Cohn LH. Minimally-invasive valve operations. J Am Coll Cardiol. 2010;56:455-62.

5. Gregoric ID, Cohn WE, Akay MH, La Francesca S, Myers T, Frazier OH. CentriMag left ventricular assist system: cannulation through a right minithoracotomy. Tex Heart Inst J. 2008;35:184-5. 